\title{
JOINT USES OF VLBI WITH ASTRONOMICAL OPTICAL INSTRUMENTS FOR STUDYING VERTICAL CHANGES
}

\author{
Li Zhi-sen, Zhang Guo-dong, and Han Yan-ben \\ Beijing Astronomical Observatory \\ Academia Sinica \\ Beijing, People's Republic of China
}

The description of the gravitational field at the surface of the Earth requires two quantities: the absolute value of the gravitational acceleration and the gravitational direction (deviation from vertical direction). At present, the various gravimeters measure the former quantity, and there is no effective instrument for monitoring the latter. This shortcoming seriously affects the comprehension and further knowledge of the gravitational field.

It is well known that VLBI can be applied to measure earth rotation parameters. VLBI observations have great precision, and the measurements are referred to the inertial frame of the radio sources. They will soon replace optical instruments and other techniques that monitor earth rotation parameters.

Astronomical optical observations in support of geodesy have a history of about 80 years. They give numerous data of angle values between the rotation's axis and the vertical (e.g., plumb line) at some places. By analysis of their residuals, we have found that the optical instruments can detect changes in the local vertical, at least the violent vertical changes that occur before major earthquakes. The Soviet astronomer N. T. Mironov has also studied the variations of the directions of plumb lines from data of astronomical observations. The plumb line at a given place not only has tidal variation (about $0 .{ }^{\prime \prime} 03$ ) but also changes due to the motions of ground mass, such as ground water before major earthquakes. These changes can exceed 0 "' 1 .

VLBI is a geometrical measurement technique, and it can be used to measure the geodetic coordinates. Therefore, its joint use and coordinated observation with optical measurements at a certain place could provide valuable data on vertical changes. For this purpose, portable VLBI equipment should be developed. 